\title{
Active pooling design in group testing based on Bayesian posterior prediction
}

\author{
Ayaka Sakata $\oplus^{*}$ \\ Institute of Statistical Mathematics, 10-3 Midori-cho, Tachikawa, Tokyo 190-8562, Japan; \\ Department of Statistical Science, The Graduate University for Advanced Science (SOKENDAI), \\ Hayama-cho, Kanagawa 240-0193, Japan; \\ and JST PRESTO, 4-1-8 Honcho, Kawaguchi, Saitama 332-0012, Japan
}

(Received 19 August 2020; revised 5 January 2021; accepted 6 January 2021; published 5 February 2021)

\begin{abstract}
For identifying infected patients in a population, group testing is an effective method to reduce the number of tests and correct test errors. In group testing, tests are performed on pools of specimens collected from patients, where the number of pools is lower than that of patients. The performance of group testing considerably depends on the design of pools and algorithms that are used for inferring the infected patients from the test outcomes. In this paper, an adaptive design method of pools based on the predictive distribution is proposed in the framework of Bayesian inference. The proposed method, executed using a belief propagation algorithm, results in more accurate identification of the infected patients compared with the group testing performed on random pools determined in advance.
\end{abstract}

DOI: 10.1103/PhysRevE.103.022110

\section{INTRODUCTION}

Identification of infected patients from a large population using clinical tests, such as blood tests and polymerase chain reaction tests, requires significant operating costs. Group testing is one of the approaches to reduce such costs by performing tests on pools of specimens obtained from patients $[1,2]$. When the fraction of infected patients in a population is sufficiently small, the infected patients can be identified from tests on pools whose number is smaller than that of the patients. Originally, group testing was developed for blood testing by Dorfman and is now applied to various fields, such as quality control in product testing [3] and multiple access communication [4].

Group testing is roughly classified into nonadaptive group testing and adaptive group testing. In nonadaptive group testing, all pools are determined in advance and fixed during all tests. In adaptive group testing, pools are designed sequentially, depending on the previous test outcomes. Dorfman's original study considered the simplest adaptive procedure, the so-called two-stage testing; here, in the first round, tests are performed on pools designed in advance, and all patients belonging to the positive pool are individually tested in the subsequent stage. A generalization of the two-stage testing is known as a binary splitting method [3,5], where the positive pool in the previous stage is split into two subpools. Tests in the subsequent stage are performed on the subpools until the infected patients are identified. Further, the splitting of

\footnotetext{
*ayaka@ism.ac.jp
}

Published by the American Physical Society under the terms of the Creative Commons Attribution 4.0 International license. Further distribution of this work must maintain attribution to the author(s) and the published article's title, journal citation, and DOI. the positive pools into several subsets larger than two sometimes reduces the number of tests required for identifying the infected patients [6]. These splitting-based methods are effective when the number of infected patients is sufficiently small. However, the splitting-based methods exhibit a limitation in the correction of false-negative results as patients in the negative pools are never tested again, even when the negative result is false.

Unlike the splitting-based design, active design of data sampling has been studied in statistics and machine learning, known as experiments design $[7,8]$, active learning $[9,10]$, and Bayesian optimization [11,12]. In these approaches, the optimal method to select training data for efficient learning is developed considering several criteria that quantify informativeness of the unknown data. The active design of data sampling improves the performance of algorithms in several fields, such as text classification [9], semisupervised learning [13], and support vector machine [14]. Active data sampling is particularly effective when data possess uncertainty due to a noisy generative process and the number of data samplings is limited. In the context of group testing, active sampling of data corresponds to the active design of pools for the subsequent stage. Further, tests, whose number should be reduced, can possess uncertainty due to noisy observation process; active sampling makes a significant contribution by addressing these issues.

The group testing problem can be regarded as a variant of the compressed sensing in signal processing [15] and sparse estimation in statistics [16]. These problems have a mathematical similarity with the physics of random systems, and statistical-physics-based studies contribute to the understanding of these problems $[17,18]$. Unlike the rigorous approach in information theory, the statistical-physics-based method currently cannot provide a precise bound evaluation, but a reasonable approximation based on physical consideration leads to an efficient computation method with reduced 


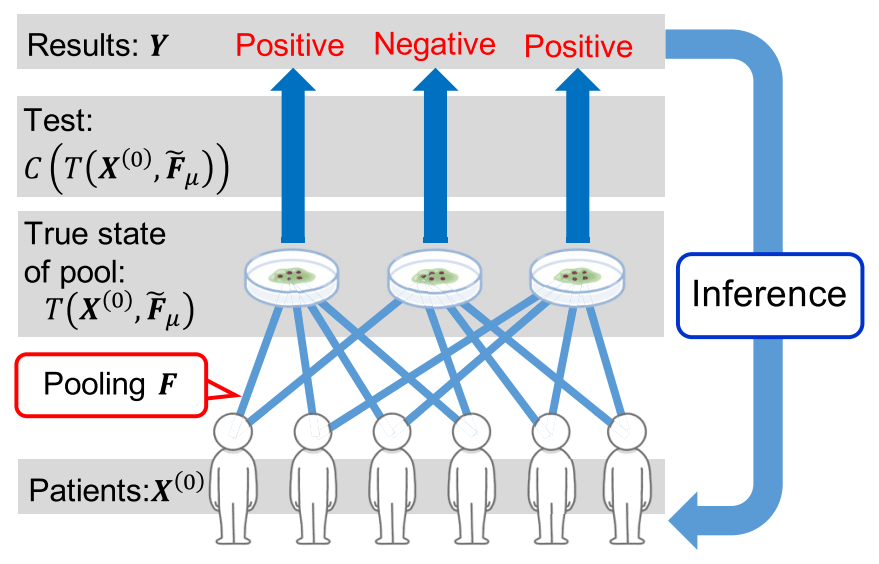

FIG. 1. Schematic picture of group testing and corresponding variables used in the mathematical formulation.

computational cost. This direction of the study is useful in situations that require a large number of clinical tests. It is natural to apply the statistical-physics-based method to the adaptive setting of group testing, which has not been studied thus far.

In this paper, we propose an active pooling design method employing Bayesian inference for efficient identification of infected patients using group testing. Bayesian modeling can consider the finite false probabilities in the test and provide a measure to quantify the uncertainty, posterior predictive distribution. We sequentially design pools based on the predictive distribution in adaptive group testing. The procedure is executed using a statistical-physics-based algorithm, belief propagation (BP) [19-22], which achieves a reasonable approximation of estimates with a feasible computational cost [23]. We demonstrate that, compared with the approach that uses randomly generated pools, the proposed pooling method effectively corrects errors with a smaller number of tests.

\section{MATHEMATICAL FORMULATION}

Figure 1 shows schematic picture of group testing considered here. Let us denote the true state of $N$ patients by $\boldsymbol{X}^{(0)} \in\{0,1\}^{N}$, where $X_{i}^{(0)}=1$ and $X_{i}^{(0)}=0$ indicate that the $i$ th patient is infected and not infected, respectively. The pooling of the patients is determined by a matrix $\boldsymbol{F} \in\{0,1\}^{M \times N}$, where $M(<N)$ is the number of pools and $F_{\mu i}=1$ and $F_{\mu i}=$ 0 indicate that the $i$ th patient is in the $\mu$ th pool and is not, respectively. The true state of the $\mu$ th group, denoted by $T\left(\boldsymbol{X}^{(0)}, \tilde{\boldsymbol{F}}_{\mu}\right)$, where $\tilde{\boldsymbol{F}}_{\mu}$ is the $\mu$ th row vector of $\boldsymbol{F}$, is given by $T\left(\boldsymbol{X}^{(0)}, \tilde{\boldsymbol{F}}_{\mu}\right)=\vee_{i=1}^{N} F_{\mu i} X_{i}^{(0)}$, where $\vee_{i=1}^{N} f_{i}=f_{1} \vee f_{2} \vee \cdots \vee$ $f_{N}$ denotes the logical sum of $N$ components. That is, when the $\mu$ th pool contains at least one infected patient, the state of the $\mu$ th pool is 1 (positive); otherwise, it is 0 (negative).

The test error is modeled by a function $C(\cdot)$ that returns 0 or 1 according to the probability conditioned by the input as

$$
\begin{aligned}
& P(C(a)=1 \mid a=1)=p_{\mathrm{TP}}, \quad P(C(a)=0 \mid a=1)=1-p_{\mathrm{TP}} \\
& P(C(a)=1 \mid a=0)=p_{\mathrm{FP}}, \quad P(C(a)=0 \mid a=0)=1-p_{\mathrm{FP}}
\end{aligned}
$$

and $p_{\mathrm{TP}}$ and $p_{\mathrm{FP}}$ correspond to the true-positive (TP) and false-positive (FP) probabilities in the test, respectively [21,23]. We assume that the test errors are independent of each other; further, from the property of $C(\cdot)$, the generative model of $\boldsymbol{Y}$ is given by $P_{\text {gen }}\left(\boldsymbol{Y} \mid \boldsymbol{X}^{(0)}, \boldsymbol{F}\right)=$ $\prod_{\mu=1}^{M} P_{\text {gen }}\left(Y_{\mu} \mid \boldsymbol{X}^{(0)}, \tilde{\boldsymbol{F}}_{\mu}\right)$, where

$$
\begin{aligned}
P_{\mathrm{gen}} & \left(Y_{\mu} \mid \boldsymbol{X}^{(0)}, \tilde{\boldsymbol{F}}_{\mu}\right) \\
= & \left\{p_{\mathrm{TP}} Y_{\mu}+\left(1-p_{\mathrm{TP}}\right)\left(1-Y_{\mu}\right)\right\} T\left(\boldsymbol{X}^{(0)}, \tilde{\boldsymbol{F}}_{\mu}\right) \\
& +\left\{p_{\mathrm{FP}} Y_{\mu}+\left(1-p_{\mathrm{FP}}\right)\left(1-Y_{\mu}\right)\right\}\left[1-T\left(\boldsymbol{X}^{(0)}, \tilde{\boldsymbol{F}}_{\mu}\right)\right]
\end{aligned}
$$

is a Bernoulli distribution conditioned by $\boldsymbol{X}$ and $\boldsymbol{F}$.

Currently, we aim to infer the true states of patients $\boldsymbol{X}^{(0)}$ from the observation $\boldsymbol{Y}$. Thus, the Bayes formula is considered. Further, we introduce the prior distribution of the patient states $P_{\text {pri }}\left(X_{i}\right) \sim \operatorname{Bernoulli}(\rho)$, where $\rho \in[0,1]$ is the assumed infection probability. Following the Bayes rule, the posterior distribution is given by $P_{\text {post }}(\boldsymbol{X} \mid \boldsymbol{Y}) \propto$ $P_{\text {gen }}(\boldsymbol{Y} \mid \boldsymbol{X}) \prod_{i} P_{\text {pri }}\left(X_{i}\right)$. The $i$ th patient's state is identified on the basis of the marginal distribution $P_{\text {post }}\left(X_{i} \mid \boldsymbol{Y}\right)=$ $\sum_{\boldsymbol{X} \backslash X_{i}} P_{\text {post }}(\boldsymbol{X} \mid \boldsymbol{Y})$, where $\boldsymbol{X} \backslash X_{i}$ denotes the components of $\boldsymbol{X}$ other than $X_{i}$. As the variable $X_{i}$ is binary, we can represent the marginal distribution using a Bernoulli probability $\theta_{i}$ as

$$
P_{\text {post }}\left(X_{i} \mid \boldsymbol{Y}\right)=\theta_{i}(\boldsymbol{Y}) X_{i}+\left[1-\theta_{i}(\boldsymbol{Y})\right]\left(1-X_{i}\right),
$$

and $\theta_{i}(\boldsymbol{Y})$ corresponds to the infection probability estimated under the test result $\boldsymbol{Y}$, namely, the probability that $X_{i}=1$. We have to convert the returned probability to a binary value for the identification of the patients' states. The simplest estimate of $X_{i}^{(0)}$ is the maximum a posteriori (MAP) estimator given by

$$
X_{i}^{(\mathrm{MAP})}=\mathbb{I}\left(\theta_{i}>0.5\right),
$$

where $\mathbb{I}(a)$ is the indicator function whose value is 1 when $a$ is true, and 0 otherwise.

\section{ADAPTIVE DESIGN OF POOLS}

Here, we divide $M$ tests into $M_{\text {ini }}$ tests under pools fixed in advance as the initial stage and $M_{\text {ada }}$ tests sequentially performed on actively designed pools as the adaptive stage. Hence, $M=M_{\text {ini }}+M_{\text {ada }}$. We denote the index set of patients who are in the $v$ th pool as $\pi(v)$, where $F_{v i}=1$ for $i \in \pi(v)$; otherwise, 0 . We consider the determination of $\pi(v+1)(v \geqslant$ $M_{\text {ini }}$ ) among possible pools denoted by $\mathcal{P}$ based on the $1, \ldots, v$ th test outcomes, denoted by $\boldsymbol{Y}_{(v)}=\left[Y_{1}, Y_{2}, \ldots, Y_{v}\right]^{\mathrm{T}}$, which are performed on pools $\pi(1), \ldots, \pi(v)$. Figure 2 shows a schematic picture of the adaptive determination of $\pi(3)$ according to the inference under the test results performed on pools $\pi(1)$ and $\pi(2)$.

Let us introduce the predictive distribution for the unknown result of the test $Y \in\{0,1\}$, which will be performed on a certain pool $\pi \in \mathcal{P}$, defined as

$$
P_{\text {pre }}\left(Y \mid \boldsymbol{Y}_{(v)}, \pi\right)=\sum_{\boldsymbol{X}_{\pi}} P_{\text {gen }}\left(Y \mid \boldsymbol{X}_{\pi}\right) P_{\text {post }}\left(\boldsymbol{X}_{\pi} \mid \boldsymbol{Y}_{(v)}\right),
$$



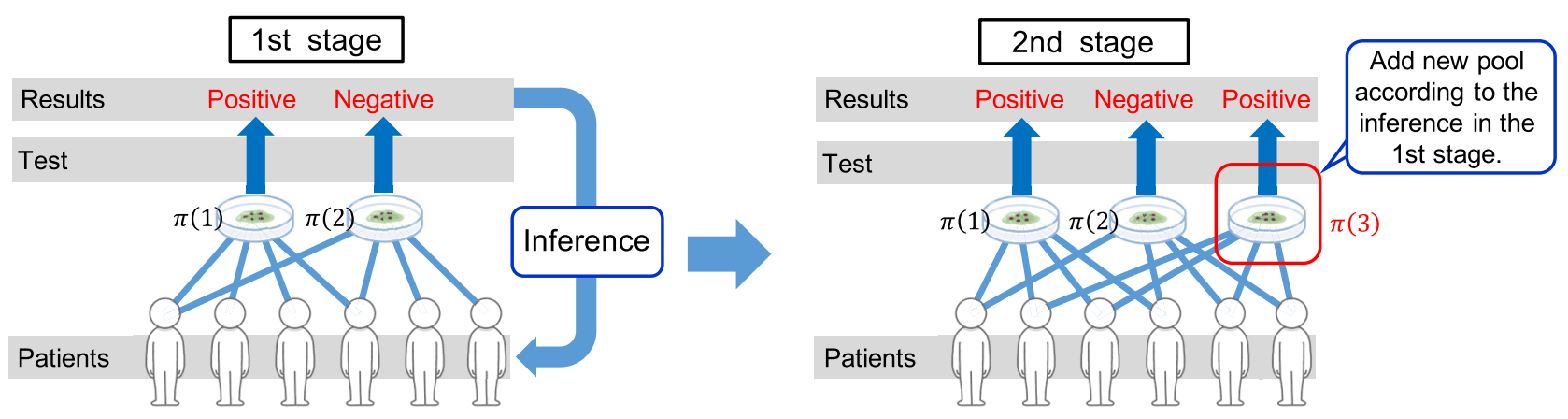

FIG. 2. Schematic picture of adaptive group testing considered here. For simplicity, the addition of one pool, $\pi$ (3), is shown.

where $P_{\text {post }}\left(\boldsymbol{X}_{\pi} \mid \boldsymbol{Y}_{(v)}\right)=\sum_{\boldsymbol{X} \backslash \boldsymbol{X}_{\pi}} P_{\text {post }}\left(\boldsymbol{X} \mid \boldsymbol{Y}_{(\nu)}\right)$ and $\quad \boldsymbol{X}_{\pi}=$ $\left\{X_{i} \mid i \in \pi\right\}$. The predictive distribution can be regarded as an approximation of the true generative process of the test output performed on $\pi$ based on the posterior at step $v$ [24]. We use the predictive distribution for active design of pools aiming for efficient reduction of "uncertainty" in decision. For intuitive discussion, let us consider the case that $P_{\text {pre }}\left(Y=1 \mid \boldsymbol{Y}_{(\nu)}, \pi\right)$ and $P_{\text {pre }}\left(Y=0 \mid \boldsymbol{Y}_{(v)}, \pi\right)$ are significantly different, namely, the predictive distribution resembles the true generative process where $p_{\mathrm{TP}}$ and $p_{\mathrm{FP}}$ are significantly different. For simplicity, we consider the case that $P_{\text {pre }}\left(Y=1 \mid \boldsymbol{Y}_{(v)}, \pi\right)$ is larger than $P_{\text {pre }}\left(Y=0 \mid \boldsymbol{Y}_{(v)}, \pi\right)$, as shown in Fig. 3(a). This means that the posterior distribution is consistent with the new observation performed on the pool $\pi$ in the sense that the current posterior matches the new test result $Y=1$, and $Y=0$ is supposed to be the test error. We do not consider this "explainable pool" in the subsequent stage because the states of the patients in the pool are already described by the current posterior, and further observation is not needed for decision. Instead, we consider "nonexplainable pool" $\pi$ that gives comparable $P_{\text {pre }}\left(Y=1 \mid \boldsymbol{Y}_{(v)}, \pi\right)$ and $P_{\text {pre }}\left(Y=0 \mid \boldsymbol{Y}_{(v)}, \pi\right)$, as shown in Fig. 3(b), where the posterior at step $v$ cannot explain the test result performed on the pool $\pi$.

This strategy can be expressed by the maximization of the predictive entropy defined as

$$
S\left(\boldsymbol{Y}_{(v)}, \pi\right)=-\sum_{Y} P_{\text {pre }}\left(Y \mid \boldsymbol{Y}_{(v)}, \pi\right) \ln P_{\text {pre }}\left(Y \mid \boldsymbol{Y}_{(v)}, \pi\right),
$$

where $P_{\text {pre }}\left(Y=1 \mid \boldsymbol{Y}_{(v)}, \pi\right)=P_{\text {pre }}\left(Y=0 \mid \boldsymbol{Y}_{(v)}, \pi\right)=0.5$ gives the entropy maximum. Active design of data sampling based on the entropy maximization is known as uncertainty (a)

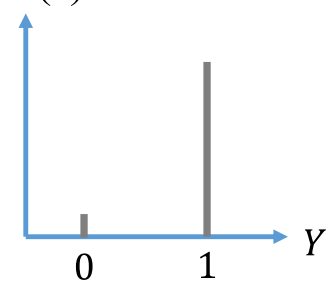

(b)

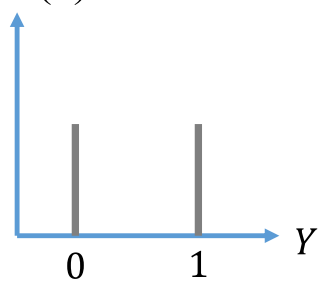

FIG. 3. Examples of predictive distribution. (a) and (b) are typical distributions for explainable pool and nonexplainable pool, respectively. sampling in active learning [25,26]. By setting $P_{\text {post }}\left(\boldsymbol{X}_{\pi}=\right.$ $\left.\mathbf{0} \mid \boldsymbol{Y}_{(v)}\right)=q\left(\pi, \boldsymbol{Y}_{(v)}\right)$, which is the estimated probability under given $\boldsymbol{Y}_{(v)}$ that all patients in the pool $\pi$ are not infected, the predictive distribution is expressed as

$$
\begin{aligned}
P_{\mathrm{pre}}( & \left.Y \mid \boldsymbol{Y}_{(\nu)}, \pi\right) \\
= & \left\{p_{\mathrm{TP}} Y+\left(1-p_{\mathrm{TP}}\right)(1-Y)\right\}\left[1-q\left(\pi, \boldsymbol{Y}_{(\nu)}\right)\right] \\
& +\left\{p_{\mathrm{FP}} Y+\left(1-p_{\mathrm{FP}}\right)(1-Y)\right\} q\left(\pi, \boldsymbol{Y}_{(\nu)}\right) .
\end{aligned}
$$

Therefore, the predictive entropy is also expressed by one parameter $q\left(\pi, \boldsymbol{Y}_{(v)}\right)$. Regarding the predictive entropy as a function of $q \in[0,1]$, the maximum of the predictive entropy is achieved at $q=q^{*}$ given by

$$
q^{*}=\frac{p_{\mathrm{TP}}-0.5}{p_{\mathrm{TP}}-p_{\mathrm{FP}}}
$$

where $p_{\mathrm{FP}}<0.5 \leqslant p_{\mathrm{TP}}$ is assumed. We determine the $(v+1)$ th pool as

$$
\pi(v+1)=\arg \min _{\pi \in \mathcal{P}}\left|q\left(\pi, \boldsymbol{Y}^{(v)}\right)-q^{*}\right| .
$$

Here, we note that the following relationship holds:

$$
\begin{aligned}
& \ln P_{\text {post }}\left(\boldsymbol{X} \mid \boldsymbol{Y}_{(v+1)}\right)-\ln P_{\text {post }}\left(\boldsymbol{X} \mid \boldsymbol{Y}_{(v)}\right) \\
& \quad=\ln P_{\text {gen }}\left(Y_{v+1} \mid \tilde{\boldsymbol{F}}_{v+1}, \boldsymbol{X}\right)-\ln P_{\text {pre }}\left(Y_{v+1} \mid \boldsymbol{Y}_{(v)}, \pi\right) .
\end{aligned}
$$

Equation (11) implies that by considering the pools whose corresponding predictive distribution differs from the true generative process, the posterior is largely modified from that in the preceding step. Therefore, the explainable pool whose predictive distribution is similar to Fig. 3(a) does not largely modify the posterior distribution. The preferential selection of the nonexplainable pool is expected to lead the efficient modification of the posterior distribution to be realistic.

The remaining task is the calculation of $q\left(\pi, \boldsymbol{Y}_{(v)}\right)$ for possible $\pi$ under the given test results $\boldsymbol{Y}_{(\nu)}$. The mathematical form of $q\left(\pi, Y_{(\nu)}\right)$ depends on the size of $\pi$, denoted by $|\pi|$. When $|\pi|=1$, we obtain

$$
q\left(\pi, \boldsymbol{Y}_{(v)}\right)=1-\theta_{\pi}\left(\boldsymbol{Y}_{(v)}\right)
$$

For larger pools, the correlation between the patients in the pool should be considered for the exact evaluation of $q\left(\pi, \boldsymbol{Y}_{(v)}\right)$. The general expression of $q\left(\pi, \boldsymbol{Y}_{(v)}\right)$ for pools 
with size $|\pi|$ is given by

$$
\begin{aligned}
q\left(\pi, \boldsymbol{Y}_{(v)}\right)= & \sum_{n=2}^{|\pi|}(-1)^{n} \sum_{\gamma \subset \pi,|\gamma|=n} \chi_{\gamma_{1} \cdots \gamma_{n}}\left(\boldsymbol{Y}_{(v)}\right) \\
& +\prod_{i=1}^{|\pi|}\left[1-\theta_{\pi_{i}}\left(\boldsymbol{Y}_{(v)}\right)\right]
\end{aligned}
$$

where $\pi_{i}(i=1, \ldots,|\pi|)$ is the $i$ th component in the pool $\pi \quad$ and $\quad \chi_{\gamma_{1} \cdots \gamma_{n}}\left(\boldsymbol{Y}_{(v)}\right) \equiv E_{\mathrm{post} \mid \boldsymbol{Y}_{(v)}}\left[\prod_{i=1}^{n} X_{\gamma_{i}}\right]-\prod_{i=1}^{n} \theta_{\gamma_{i}}\left(\boldsymbol{Y}_{(v)}\right)$, where $E_{\text {post } \mid \boldsymbol{Y}_{(v)}}[\cdot]$ denotes the average according to the posterior distribution $P_{\text {post }}\left(\boldsymbol{X} \mid \boldsymbol{Y}_{(\nu)}\right)$. For example, when $\pi=$ $\{i, j\}(|\pi|=2)$, we obtain

$$
q\left(\pi, \boldsymbol{Y}_{(v)}\right)=\chi_{i j}\left(\boldsymbol{Y}_{(v)}\right)+\left[1-\theta_{i}\left(\boldsymbol{Y}_{(v)}\right)\right]\left[1-\theta_{j}\left(\boldsymbol{Y}_{(v)}\right)\right],
$$

where $\chi_{i j}\left(\boldsymbol{Y}_{(v)}\right)=E_{\text {post } \mid \boldsymbol{Y}_{(v)}}\left[X_{i} X_{j}\right]-\theta_{i}\left(\boldsymbol{Y}_{(v)}\right) \theta_{j}\left(\boldsymbol{Y}_{(v)}\right)$ is the susceptibility.

Next, we discuss the relationship between $q^{*}, p_{\mathrm{TP}}$, and $p_{\mathrm{FP}}$. From the definition of $q^{*}$, Eq. (9), if $p_{\mathrm{TP}}>1-p_{\mathrm{FP}}$, then $q^{*}<0.5$. This indicates that the pools with $q\left(\pi, \boldsymbol{Y}_{(v)}\right)<$ 0.5 are likely to be chosen when $p_{\mathrm{TP}}>1-p_{\mathrm{FP}}$. In other words, when the probability that at least one patient in a pool is infected is larger than the probability that no one is infected, the pool tends to be chosen. This can be understood as follows. Introducing false-negative probability $p_{\mathrm{FN}}, p_{\mathrm{TP}}>$ $1-p_{\mathrm{FP}}$ is equivalent to $p_{\mathrm{FN}}<p_{\mathrm{FP}}$. This implies that false test results are mainly contained in positive results. Hence, pools with $q\left(\pi, \boldsymbol{Y}_{(v)}\right)<0.5$ contain significant uncertainty, compared with $q\left(\pi, \boldsymbol{Y}_{(v)}\right)>0.5$. Therefore, in active pooling design based on uncertainty, pools with $q\left(\pi, \boldsymbol{Y}_{(\nu)}\right)<0.5$ are preferably chosen, when $p_{\mathrm{TP}}>1-p_{\mathrm{FP}}$. Following the same logic, we can understand that the pool with $q\left(\pi, \boldsymbol{Y}_{(v)}\right)>0.5$ is likely to be chosen when $p_{\mathrm{TP}}<1-p_{\mathrm{FP}}$.

\section{IMPLEMENTATION BY BELIEF PROPAGATION}

The computation of the marginal distribution requires the exponential order of the sums and thus is intractable. We approximately calculate the marginal distribution using the $\mathrm{BP}$ algorithm [20-22]. Compared with the approximation using the BP algorithm with the exact calculation at a small size, the BP algorithm has sufficient approximation performance when applied to group testing [23]. In this study, we use the BP algorithm as a reasonable method owing to its approximation accuracy and computational time. In Appendix A, the BP algorithm for calculating the infection probability given by the posterior distribution is summarized. We denote the obtained estimates of $\theta_{i}$ and the corresponding MAP estimator as $\hat{\theta}_{i}$ and $\hat{X}_{i}^{(\mathrm{MAP})}=\mathbb{I}\left(\hat{\theta}_{i}>0.5\right)$, respectively. We measure the accuracy of the MAP estimator by the TP and FP rates given by

$$
\mathrm{TP}=\frac{\sum_{i} X_{i}^{(0)} \hat{X}_{i}^{(\mathrm{MAP})}}{\sum_{i} X_{i}^{(0)}}, \quad \mathrm{FP}=\frac{\sum_{i}\left(1-X_{i}^{(0)}\right) \hat{X}_{i}^{(\mathrm{MAP})}}{\sum_{i}\left(1-X_{i}^{(0)}\right)},
$$

respectively. A TP value larger than $p_{\mathrm{TP}}$ and a FP value smaller than $p_{\mathrm{FP}}$ indicate that the BP-based identification has better performance than the parallel test of $N$ patients.
To apply the BP algorithm to an adaptive test, we should obtain $q\left(\pi, \boldsymbol{Y}_{(v)}\right)$ for each $v\left(>M_{\text {ini }}\right)$. For its exact computation, we need multibody correlations between patients except when $|\pi|=1$, which is expressed as the first term of Eq. (13), although the BP algorithm returns one-body information. In this study, we use the simplest approximation provided by the BP algorithm as $\hat{q}\left(\pi, \boldsymbol{Y}_{(v)}\right) \equiv \prod_{i=1}^{|\pi|}\left(1-\hat{\theta}_{\pi_{i}}\right)$ to avoid the increase in the computational time required for the calculation of multibody correlation. In principle, BP can approximately compute the correlation between patients by deriving conditional posterior expectations, which requires additional computations of the order of $O[N ! /(N-|\pi|) !]$ according to the product rule of conditional joint distributions. As an example, we calculate the susceptibility using the BP algorithm and implement active pooling design on the basis of $q\left(\pi, \boldsymbol{Y}^{(v)}\right)$ for the $|\pi|=2$ case, as shown in Appendix B. The consideration of the susceptibility does not provide large improvements in terms of TP and FP rates in the problem setting studied herein. Hence, we use one-body approximation $\hat{q}\left(\pi, \boldsymbol{Y}_{(v)}\right)$ throughout the study.

Further, to reduce the computational time of $q\left(\pi, \boldsymbol{Y}^{(v)}\right)$ for all possible $\pi$, we focus on the subspace of pools $\mathcal{P}_{1}=$ $\{\pi|| \pi \mid=1, \pi \in \mathcal{P}\}$ and $\mathcal{P}_{2}=\{\pi|| \pi \mid \leqslant 2, \pi \in \mathcal{P}\}$; hence, $\mathcal{P}_{1} \subset \mathcal{P}_{2} \subset \mathcal{P}$. In general, the number of the pools with size $|\pi| \sim O(1)$ is $O\left(N^{|\pi|}\right)$. Therefore, for the determination of the pool according to Eq. (10), $q\left(\pi, \boldsymbol{Y}^{(v)}\right)$ should be computed $O\left(N^{|\pi|}\right)$ times corresponding to each pool. Mathematically, the restriction on the pooling space can prevent the sampling of the most uncertain pool. However, it leads to the drastic reduction in the computational cost required for the determination of the pool. In the case of $\mathcal{P}_{2}$, the computational cost is $O\left(N^{2}\right)$, which is considered acceptable in practical implementations. Furthermore, in the settings of the numerical simulations, the performance of the adaptive procedure is not so much improved by considering the pools of $|\pi|>2$. As an example, TP and FP considering the pooling space $\mathcal{P}_{3}=\{\pi|| \pi \mid \leqslant 3, \pi \in \mathcal{P}\}$ is compared with that under $\mathcal{P}_{1}$ and $\mathcal{P}_{2}$ in Appendix C.

The setting of the numerical simulation described in this section is as follows. Let us denote the longitudinal coupling of matrices or vectors $\boldsymbol{a}$ and $\boldsymbol{b}$ that have the same number of columns as $[\boldsymbol{a} ; \boldsymbol{b}]$. Hence, $\boldsymbol{F}=\left[\tilde{\boldsymbol{F}}_{1} ; \tilde{\boldsymbol{F}}_{2} ; \cdots ; \tilde{\boldsymbol{F}}_{M}\right]$. The submatrix of $\boldsymbol{F}$ given from the 1st to the $\nu$ th row vectors is denoted by $\boldsymbol{F}_{(v)}=\left[\tilde{\boldsymbol{F}}_{1} ; \ldots ; \tilde{\boldsymbol{F}}_{v}\right]$; hence, $\boldsymbol{F}_{(v+1)}=\left[\boldsymbol{F}_{(v)} ; \tilde{\boldsymbol{F}}_{v+1}\right]$. The pooling matrix for the initial stage, $\boldsymbol{F}_{\left(M_{\mathrm{ini}}\right)}$, is randomly generated under the constraint that the number of pools each patient belongs to and the number of patients in each pool are fixed at $N_{G}(\ll N)$ and $N_{O}(\ll N)$, respectively. Hence, $\sum_{i=1}^{N} F_{\mu i}=N_{G}$ for $\mu \leqslant M_{\text {ini }}$ and $\sum_{\mu=1}^{M_{\text {ini }}} F_{\mu i}=N_{O} \forall i$ hold, and the relationship $N_{O}=N_{G} M_{\text {ini }} / N$ holds. The corresponding test result in the initial stage, $\boldsymbol{Y}_{\left(M_{\text {ini }}\right)}$, is generated as $\boldsymbol{Y}_{\left(M_{\text {ini }}\right)} \sim$ $P_{\text {gen }}\left(\boldsymbol{Y} \mid \boldsymbol{X}^{(0)}, \boldsymbol{F}_{\left(M_{\text {ini }}\right)}\right)$. The posterior distribution under given $\boldsymbol{Y}_{\left(M_{\text {ini }}\right)}$ and $\boldsymbol{F}_{\left(M_{\text {ini }}\right)}$ is approximately calculated using the BP algorithm. For the subsequent adaptive stage, we actively choose $\pi\left(M_{\text {ini }}+1\right)$ among $\mathcal{P}_{1}$ or $\mathcal{P}_{2}$ based on the predictive entropy given by the posterior distribution of the initial stage. Next, we construct $\widetilde{\boldsymbol{F}}_{M_{\text {ini }}+1}$ to ensure that $F_{M_{\text {ini }}+1, i}=1$ for $i \in \pi\left(M_{\text {ini }}+1\right)$; otherwise, 0 . The test result is generated as $Y_{M_{\mathrm{ini}}+1} \sim P_{\mathrm{gen}}\left(Y \mid \boldsymbol{X}^{(0)}, \tilde{\boldsymbol{F}}_{M_{\mathrm{ini}}+1}\right)$, and we obtain the posterior 
Algorithm 1 Group testing with active pooling design using the belief propagation (BP) algorithm.

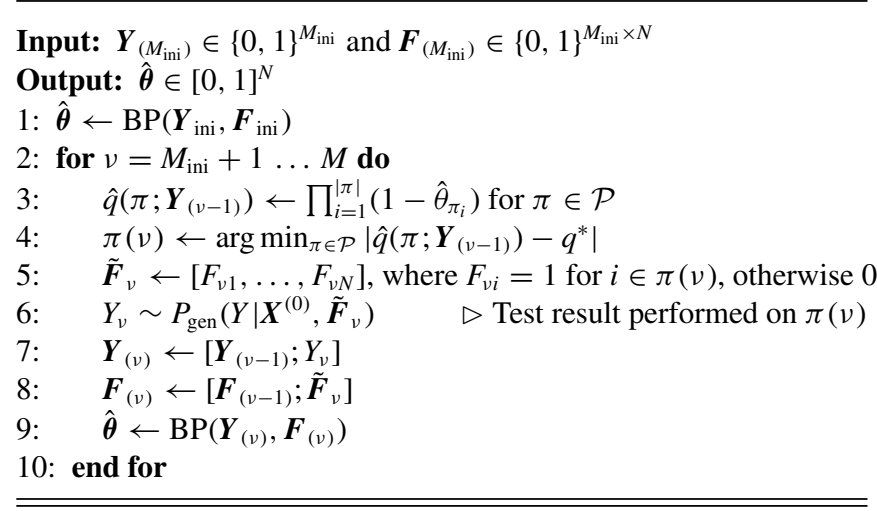

distribution under $\boldsymbol{F}_{\left(M_{\mathrm{ini}}+1\right)}=\left[\boldsymbol{F}_{\left(M_{\mathrm{ini}}\right)} ; \tilde{\boldsymbol{F}}_{M_{\mathrm{ini}}+1}\right]$ and $\boldsymbol{Y}_{\left(M_{\mathrm{ini}}+1\right)}=$ $\left[\boldsymbol{Y}_{\left(M_{\mathrm{ini}}\right)} ; Y_{M_{\text {ini }}+1}\right]$ using the BP algorithm. This adaptive test procedure is repeated $M_{\text {ada }}$ times, where $M=M_{\text {ini }}+M_{\text {ada }}$, and the state of patients is determined by the MAP estimator corresponding to $\hat{\theta}(\boldsymbol{Y}, \boldsymbol{F})$, where $\boldsymbol{Y}=\left[\boldsymbol{Y}_{\left(M_{\mathrm{ini}}\right)} ; Y_{M_{\mathrm{ini}+1}} ; \ldots ; Y_{M}\right]$ and $\boldsymbol{F}=\left[\boldsymbol{F}_{\left(M_{\mathrm{ini}}\right)} ; \tilde{\boldsymbol{F}}_{M_{\mathrm{ini}}+1} ; \ldots ; \tilde{\boldsymbol{F}}_{M}\right]$. The pseudocode is summarized in Algorithm 1, where $\operatorname{BP}(\boldsymbol{Y}, \boldsymbol{F})$ indicates the calculation of the infection probability using the BP algorithm under the input $\boldsymbol{Y}$ and $\boldsymbol{F}$ (see Appendix A).

The true state of patients $\boldsymbol{X}^{(0)}$ is randomly generated under the constraint that $\sum_{i} X_{i}^{(0)}=N \rho$. Here, we assume that the correct parameters $\rho, p_{\mathrm{TP}}$, and $p_{\mathrm{FP}}$ are known in advance. For more general cases where the estimation of unknown parameters is required, we can construct their estimators by combining the $\mathrm{BP}$ algorithm with the expectation-maximization method or by introducing a hierarchical Bayes model [23].

Figure 4 shows the $\rho$ dependence of (a) TP and (b) FP at $N=1000, M=400$ with $M_{\text {ini }}=300$ and $M_{\text {ada }}=100$. The error probabilities are set to $p_{\mathrm{TP}}=0.9$ and $p_{\mathrm{FP}}=0.05$, and the group size in the initial stage is $N_{G}=10 . P_{1}$ and $P_{2}$ in the figure denote the results of the active pooling in the spaces $\mathcal{P}_{1}$ and $\mathcal{P}_{2}$, respectively [27]. For comparison, the results of random pooling are shown, where tests in $M_{\text {ada }}$
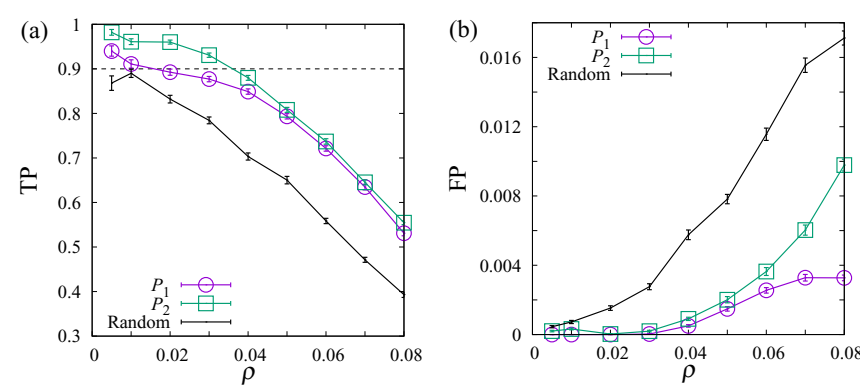

FIG. 4. $\rho$ dependence of (a) TP and (b) FP at $N=1000$ and $M=$ 400, where $M_{\text {ini }}=300$ and $M_{\text {ada }}=100$. In the initial stage, random pooling with $N_{G}=10$ is used. The error rates are fixed at $p_{\mathrm{TP}}=0.9$ and $p_{\mathrm{FP}}=0.05$. The horizontal dashed line in (a) represents $p_{\mathrm{TP}}$. For comparison, results of a random test with $M=400$ pools is shown. $P_{1}$ and $P_{2}$ denote $\mathcal{P}_{1}$ and $\mathcal{P}_{2}$ cases, respectively.
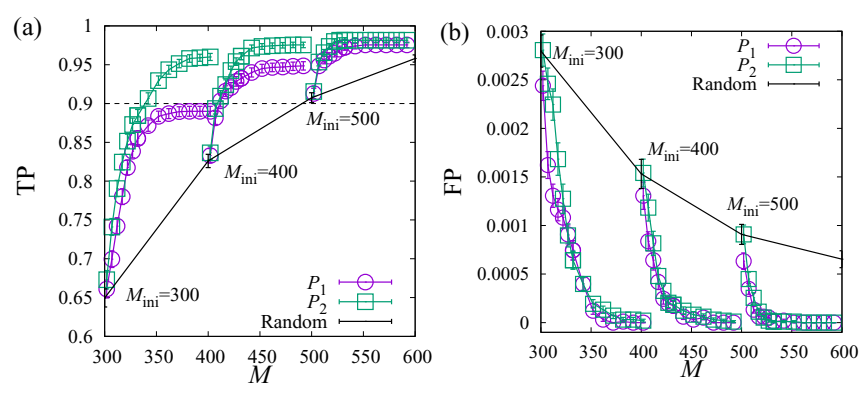

FIG. 5. $M$ dependence of (a) TP and (b) FP at $N=1000$ and $\rho=0.02$. The adaptive tests are performed 100 times after $M_{\text {ini }}=$ 300,400 , and 500 random tests with $N_{G}=10$. The error probabilities on the test are fixed at $p_{\mathrm{TP}}=0.9$ and $p_{\mathrm{FP}}=0.05$. The horizontal dashed line in (a) represents $p_{\text {TP }}$.

steps are performed on random pools generated by the same rule as the initial $M_{\text {ini-times tests. Each data point represents }}$ the averaged value with respect to 100 realizations of $\boldsymbol{Y}_{\left(M_{\mathrm{ini}}\right)}$, $\boldsymbol{F}_{\left(M_{\mathrm{ini}}\right)}$, and $\boldsymbol{X}^{(0)}$. For any region of $\rho$, TP under a random test cannot exceed the $p_{\mathrm{TP}}$, which is indicated by the horizontal line in Fig. 4(a). The adaptive test improves TP and achieves TP $>p_{\text {TP }}$ when $\rho<0.02$ for the $\mathcal{P}_{1}$ case and $\rho<0.04$ for the $\mathcal{P}_{2}$ case. As shown in Fig. 4(b), FP is smaller than $p_{\text {FP }}$ even when the pooling is randomly determined, but the adaptive test can further decrease FP.

The performance of the adaptive test depends on the number of initial random tests $M_{\text {ini }}$. Figure 5 shows the $M_{\text {ini }}$ dependence of (a) TP and (b) FP at $N=1000, \rho=0.02$, $p_{\mathrm{FP}}=0.9$, and $p_{\mathrm{TP}}=0.05$. The pool size at the initial stage is $N_{G}=10$. The figure also presents the results for $M_{\text {ini }}=300$, 400, and 500. The horizontal dashed line in (a) represents the TP probability of the test, which is 0.9 . As $M_{\text {ini }}$ increases, a high TP close to 1 is obtained via the adaptive test. Moreover, for a large $M_{\text {ini }}$, such as $M_{\text {ini }}=500$, the possible pooling space does not significantly influence the performance in terms of TP and FP. Meanwhile, for a small $M_{\text {ini }}$, the result of TP depends on the pooling space, and more accurate identification is achieved by $\mathcal{P}_{2}$. The test results at the initial stage have large uncertainties when $M_{\text {ini }}$ is small; hence, it is considered that the large pooling space is required for the effective sampling of the uncertain pools.

As shown in Fig. 5(a), to achieve a high TP, the active pooling method requires a smaller number of tests than that required by the random pooling method. For instance, the active pooling in $\mathcal{P}_{2}$ with $M_{\text {ini }}=300$ initial stage results in a high TP $>p_{\mathrm{TP}}$ after the $M_{\text {ada }}=40$ adaptive stage, namely, $M=340$. Meanwhile, the random pooling achieves TP $>p_{\mathrm{TP}}$ with almost $M=500$ tests. Regarding the improvement of $\mathrm{TP}$, the adaptive method helps effectively identify infected patients with a small number of tests.

The active pooling is robust to the errors in the test compared with the random pooling. Figure 6 shows (a) the $p_{\mathrm{TP}}$ dependence of TP for $p_{\mathrm{FP}}=0.05$ and (b) the $p_{\mathrm{FP}}$ dependence of TP for $p_{\mathrm{TP}}=0.95$ at $N=1000, M_{\text {ini }}=300, M_{\text {ada }}=100$, and $\rho=0.02$. The random tests in the initial stage are performed on pools of size $N_{G}=10$. For the random pooling case, TP $>p_{\mathrm{TP}}$ is achieved only when $p_{\mathrm{FP}}$ is sufficiently small, such as $p_{\mathrm{FP}}<0.02$. The adaptive test improves TP, and 

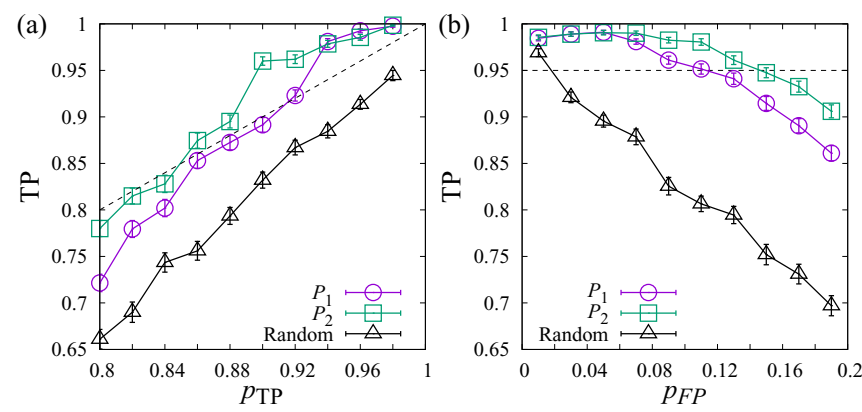

FIG. 6. $p_{\mathrm{TP}}$ dependence of TP for (a) $p_{\mathrm{FP}}=0.05$ and (b) $p_{\mathrm{TP}}=$ 0.95 at $N=1000$ and $\rho=0.02$. The adaptive tests are conducted 100 times after $M_{\text {ini }}=300$; hence, $M=400$ in total. The pool size in the random tests is $N_{G}=10$. The horizontal dashed lines represent $\mathrm{TP}=p_{\mathrm{TP}}$.

the parameter region where $\mathrm{TP}>p_{\mathrm{TP}}$ is extended in particular for the case $\mathcal{P}_{2}$.

These results indicate the efficiency of the active pooling design based on predictive distribution in group testing. However, a limitation of this approach is that the computational cost involved is higher than that of the nonadaptive approach. The estimation of the infection probability via the BP algorithm $M_{\text {ada }}$ steps is obtained again; hence, the computational cost of the adaptive approach is approximately $M_{\text {ada }}$ times larger than that of the nonadaptive approach. However, the adaptive approach achieves accurate estimation using a small number of tests. The tradeoff between the reduction of the operating cost involved in tests and the increase in the computation time of inference should be considered for practical application of the adaptive approach.

\section{SUMMARY AND DISCUSSION}

In this paper, we proposed an active pooling design in adaptive group testing, where the pool for the subsequent stage is determined based on the Bayesian posterior predictive distribution under the test outcomes in the previous stage. The proposed method was implemented using the BP algorithm, and the identification of infected patients using adaptive tests was demonstrated to be more accurate than that using randomly designed pools. In particular, the active pooling design reduced the number of required tests to achieve TP $>p_{\mathrm{TP}}$. Further, the proposed method is robust to test errors and $\mathrm{TP}>p_{\mathrm{TP}}$ holds in smaller $p_{\mathrm{TP}}$ and larger $p_{\mathrm{FP}}$, compared with the approach that uses randomly designed pools.

In the current study, we restrict the possible pooling space within $\mathcal{P}_{1}$ and $\mathcal{P}_{2}$. Mathematically, more uncertain pools can be considered removing this restriction, and further improvement in the TP and FP rates is expected. However, the straightforward calculation of the predictive entropy for all possible $\pi$ is computationally intractable. Hence, some approximation will be required. An efficient sampling method in $\pi \in \mathcal{P}$ to find the uncertain pool should be developed.

We focused on the MAP estimator to convert the estimated infection probability, which is $[0,1]$ variable, into the state of patients, $\{0,1\}$ variable, because of its simplicity; however, changing the decision threshold from 0.5 resulted in improvements in the TP rate. For example, the estimate using the confidence interval constructed based on the bootstrap method was obtained. Further, the TP rate obtained using this method was higher than that obtained using the MAP estimator [23]; however, its computational cost is unrealistic to accompany the active pooling procedure. The receiver operating characteristic (ROC) analysis is a promising method to understand the appropriate decision threshold [28,29]. Along with the ROC analysis, the quantitative background of the active pooling proposed in this paper is expected to be established.

\section{ACKNOWLEDGMENTS}

This work was accomplished thanks to the author's pleasant discussions with Yukito Iba. Further, the author thanks Koji Hukushima, Yoshiyuki Kabashima, and Satoshi Takabe for their helpful comments and discussions. This research was partially supported by Grant-in-Aid for Scientific Research 19K20363 from the Japanese Society for the Promotion of Science (JSPS), Japan Science and Technology Agency (JST) PRESTO Grant No. JPMJPR19M2, Japan, and CASIO Science Promotion Foundation.

\section{APPENDIX A: BP ALGORITHM FOR GROUP TESTING}

We denote $\pi(\mu)$ and $\mathcal{G}(i)$ as the indices of the patients in the $\mu$ th pool and those of the pools in which the $i$ th patient is included, respectively. For the edge that connects the $\mu$ th factor (test) and the $i$ th variable (patient), two types of messages $m_{i \rightarrow \mu}\left(X_{i}\right)$ and $\tilde{m}_{\mu \rightarrow i}\left(X_{i}\right)$ are defined. Intuitively, the messages $m_{i \rightarrow \mu}\left(X_{i}\right)$ and $\tilde{m}_{\mu \rightarrow i}\left(X_{i}\right)$ represent the marginal distributions of $X_{i}$ before and after the $\mu$ th test is performed, respectively. The variable $X_{i}$ is binary. Hence, the messages can be expressed by the Bernoulli probability $\theta_{i \rightarrow \mu}$ and $\tilde{\theta}_{\mu \rightarrow i}$ given by

$$
\theta_{i \rightarrow \mu}=\frac{\rho \prod_{v \in \mathcal{G}(i) \backslash \mu} \tilde{\theta}_{\nu \rightarrow i}}{Z_{i \rightarrow \mu}}, \quad \tilde{\theta}_{\mu \rightarrow i}=\frac{U_{\mu}}{\tilde{Z}_{\mu \rightarrow i}},
$$

where $\quad U_{\mu}=p_{\mathrm{TP}} Y_{\mu}+\left(1-p_{\mathrm{TP}}\right)\left(1-Y_{\mu}\right), \quad W_{\mu}=p_{\mathrm{FP}} Y_{\mu}+$ $\left(1-p_{\mathrm{FP}}\right)\left(1-Y_{\mu}\right)$ and

$$
\begin{aligned}
& \tilde{Z}_{\mu \rightarrow i}=U_{\mu}\left(2-\prod_{j \in \pi(\mu) \backslash i}\left(1-\theta_{j \rightarrow \mu}\right)\right)+W_{\mu} \prod_{j \in \pi(\mu) \backslash i}\left(1-\theta_{j \rightarrow \mu}\right), \\
& Z_{i \rightarrow \mu}=\rho \prod_{\nu \in \mathcal{G}(i) \backslash \mu} \tilde{\theta}_{\nu \rightarrow i}+(1-\rho) \prod_{\nu \in \mathcal{G}(i) \backslash \mu}\left(1-\tilde{\theta}_{\nu \rightarrow i}\right) . \quad \text { (A2) }
\end{aligned}
$$

The BP algorithm consists of the recursive update of $\theta_{i \rightarrow \mu}$ and $\tilde{\theta}_{\mu \rightarrow i}$, and at the fixed point, the infection probability is given by $[20,21,23]$

$$
\hat{\theta}_{i}=\frac{\rho \prod_{\mu \in \mathcal{G}(i)} \tilde{\theta}_{\mu \rightarrow i}}{\rho \prod_{\mu \in \mathcal{G}(i)} \tilde{\theta}_{\mu \rightarrow i}+(1-\rho) \prod_{\mu \in \mathcal{G}(i)}\left(1-\tilde{\theta}_{\mu \rightarrow i}\right)} .
$$

\section{APPENDIX B: CALCULATION OF SUSCEPTIBILITY USING THE BP ALGORITHM}

A widely used method to calculate susceptibility in the framework of the BP algorithm is susceptibility 

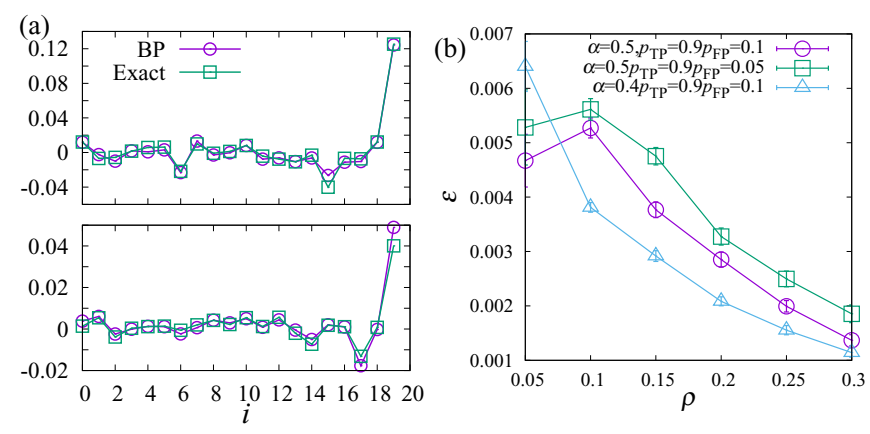

FIG. 7. (a) Examples of susceptibility calculated using the BP algorithm and exact computation at $N=20, M=10, N_{G}=10, N_{O}=$ $5, \rho=0.1, p_{\mathrm{TP}}=0.95$, and $p_{\mathrm{FP}}=0.05 . \chi_{i j}$ for $j=20$ is shown for two different realizations of $\boldsymbol{Y}, \boldsymbol{F}$, and $\boldsymbol{X}^{(0)}$. (b) Quantification of the difference between susceptibilities given by the BP algorithm and the exact calculations at $N=20$ and $N_{G}=10$ for several parameters using $\epsilon$.

propagation [30,31], where recursive update of tensors that provide susceptibility is introduced on the basis of linearresponse theory. In the current problem setting, the variables to be estimated obey the Bernoulli probability. Hence, we can compute the susceptibility in a simpler way.

Let us denote the expectation of $X_{j}$ under the posterior conditional distribution $\sum_{\boldsymbol{X} \backslash i, j} P_{\text {post }}\left(X_{j}, X_{i}=1, \boldsymbol{X}_{\backslash i, j} \mid \boldsymbol{Y}\right)$ as $\theta_{j}^{\mid X_{i}=1} \equiv E_{\mathrm{post} \mid Y}\left[X_{j} \mid X_{i}=1\right](i \neq j)$. This expectation value is evaluated using the BP algorithm by fixing $\theta_{i \rightarrow \mu}=1$ and $\tilde{\theta}_{\mu \rightarrow i}=1$ for $\mu \in \mathcal{G}(i)$. The conditional expectation value obtained using the BP algorithm is denoted as $\hat{\theta}_{j}^{\mid X_{i}=1}$. Thus, the susceptibility is given by $\hat{\chi}_{i j}=\hat{\theta}_{i} \hat{\theta}_{j}^{\mid X_{i}=1}-\hat{\theta}_{i} \hat{\theta}_{j}$.

To verify the accuracy of the susceptibility derived using the BP algorithm, we compute the exact posterior distribution by sampling all configurations in $\{0,1\}^{N}$. Examples of the exact susceptibility and the approximated one are shown in Fig. 7(a) at $N=20, M=10, \rho=0.1, p_{\mathrm{TP}}=0.95$, and $p_{\mathrm{FP}}=0.05$, where the $i$ dependence of $\chi_{i, 20}$ is shown for two different realizations of $\boldsymbol{Y}, \boldsymbol{F}$, and $\boldsymbol{X}^{(0)}$. Here, the pooling matrix is randomly generated to be $N_{G}=10$ and $N_{O}=5$. The
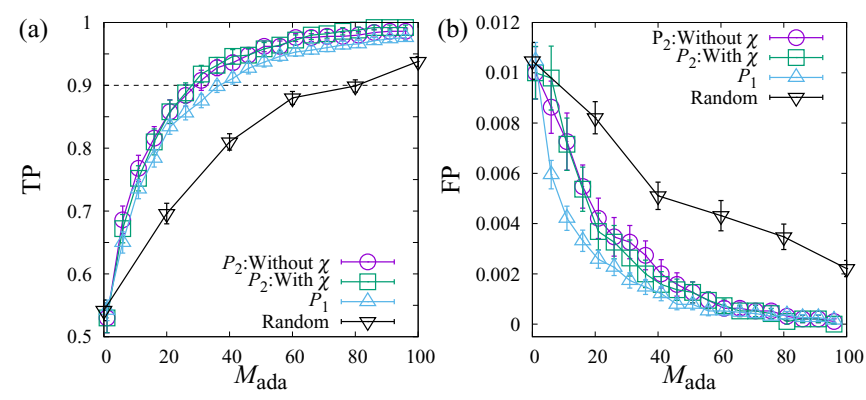

FIG. 8. Comparison of (a) TP and (b) FP for the $\mathcal{P}_{2}$ case considering the susceptibility, the $\mathcal{P}_{2}$ case without considering the susceptibility, and the $\mathcal{P}_{1}$ case, at $N=200, M_{\text {ini }}=80, \rho=0.05$, $p_{\mathrm{TP}}=0.9$, and $p_{\mathrm{FP}}=0.1$. The tests in the initial stage are performed on the randomly designed pools with $N_{G}=10$ and $N_{O}=4$.
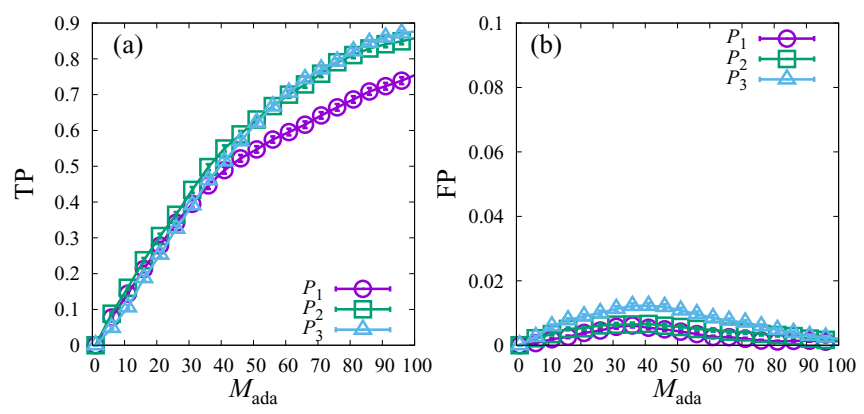

FIG. 9. Comparison of (a) TP and (b) FP for $\mathcal{P}_{1}, \mathcal{P}_{2}$, and $\mathcal{P}_{3}$ at $N=200, M_{\text {ini }}=40 . \rho=0.1, p_{\mathrm{TP}}=0.9$, and $p_{\mathrm{FP}}=0.05$.

difference between $\chi$ and $\hat{\chi}$ is quantified by $\epsilon \equiv \sum_{i<j}\left(\chi_{i j}-\right.$ $\left.\hat{\chi}_{i j}\right)^{2} /\{N(N-1) / 2\}$, whose behavior is shown in Fig. 7(b) at $N=20$ and different values of $\alpha \equiv M / N, p_{\mathrm{TP}}$, and $p_{\mathrm{FP}}$. For any parameter region, $\epsilon$ is $O\left(10^{-3}\right)$. Therefore, we consider that the $\mathrm{BP}$ algorithm provides a reasonable approximation of the susceptibility and expect that it is also applicable for larger $N$.

In Fig. 8, (a) TP and (b) FP are shown for the cases when the susceptibility is considered (denoted by " $P_{2}$ : with $\chi$ ") and not considered (denoted by " $P_{2}$ : without $\chi$ "); that is, Eq. (14) is used to determine the pool in the subsequent stage by substituting $\hat{\chi}$ calculated by BP into $\chi$ at $N=200, \rho=0.05$, $p_{\mathrm{TP}}=0.9$, and $p_{\mathrm{FP}}=0.1$. Each data point is averaged over 50 samples of $\boldsymbol{F}, \boldsymbol{X}^{(0)}$, and $\boldsymbol{Y}$. The initial stage comprises $M_{\text {ini }}=80$ random tests with $N_{G}=10$ and $N_{O}=4$. Considering the susceptibility, a slight improvement in TP is observed.

Following the same procedure, we can compute a higher order correlation in principle. For example, $E_{\text {post } \mid \boldsymbol{Y}}\left[X_{i} \mid X_{j}=\right.$ $\left.1, X_{k}=1\right]$ is obtained by fixing $\theta_{j \rightarrow \mu}=1, \theta_{k \rightarrow v}=1$, and $\tilde{\theta}_{\mu \rightarrow j}=1, \tilde{\theta}_{\nu \rightarrow k}=1$ for $\mu \in \mathcal{G}(j)$ and $v \in \mathcal{G}(k)$.

\section{APPENDIX C: PERFORMANCE OF THE ADAPTIVE PROCEDURE UNDER THE POOL SPACE $\mathcal{P}_{3}$}

Figure 9 shows (a) TP and (b) FP at $N=200$ and $\rho=0.1$, and test error is characterized by $p_{\mathrm{TP}}=0.9$ and $p_{\mathrm{FP}}=0.05$. under the pooling space $\mathcal{P}_{1}, \mathcal{P}_{2}$ and $\mathcal{P}_{3}$. Here, $M_{\text {ini }}=40$ times random tests with $N_{G}=10$ are performed initially, and then the adaptive testing is performed $M_{\text {ada }}$ times. As shown in the figure, the extension of the pooling space from $\mathcal{P}_{1}$ to $\mathcal{P}_{2}$ induces the increase of TP by 0.1 after 100 times adaptive procedure. Meanwhile, the extension of the pooling space from $\mathcal{P}_{2}$ to $\mathcal{P}_{3}$ induces the increase of TP by no more than 0.01 . In fact, this small increase of TP can be achieved by continuing the adaptive procedure beyond 100 steps with $|\pi|=2$; hence, there is no need to introduce pools with $|\pi|=3$ sacrificing the computational time. Of course, in other problem settings, there is a possibility that larger pools achieve drastic improvements. The pooling space should be carefully determined for each problem setting by checking the dependency of the performance on the pooling space. 
[1] R. Dorfman, Ann. Math. Statist. 14, 436 (1943).

[2] D.-Z. Du and F. K. Hwang, Combinatorial Group Testing and Its Applications (World Scientific, Singapore, 2000).

[3] M. Sobel and P. A. Groll, Bell Labs Tech. J. 38, 1179 (1959).

[4] J. K. Wolf, IEEE Trans. Inf. Theory 31, 185 (1985).

[5] M. Sobel and P. A. Groll, Technometrics 8, 631 (1966).

[6] F. K. Hwang, J. Am. Stat. Assoc. 67, 605 (1972).

[7] V. V. Fedorov, Theory of Optimal Experiments (Academic, New York, 1972).

[8] F. Pukelsheim, Optimal Design of Experiments (Academic, New York, 1972).

[9] D. A. Cohn, Z. Ghahramani, and M. I. Jordan, J. Artif. Intell. Res. 4, 129 (1996).

[10] B. Settles, Active learning literature survey, Technical Report, University of Wisconsin-Madison, Department of Computer Sciences, 2009.

[11] E. Brochu, V. M. Cora, and N. de Freitas, arXiv:1012.2599 (2010).

[12] B. Shahriari, K. Swersky, Z. Wang, P. A. R, and N. De Freitas, Proc. IEEE 104, 148 (2015).

[13] X. Zhu, J. Lafferty, and Z. Ghahramani, in ICML-2003 Workshop on the Continuum from Labeled to Unlabeled Data (ML Research Press, 2003), pp. 58-65.

[14] S. Tong and D. Koller, J. Mach. Learning Res. 2, 45 (2001).

[15] E. J. Candes and T. Tao, IEEE Trans. Inf. Theory 51, 4203 (2005).

[16] T. Hastie, R. Tibshirani, and M. Wainwright, Statistical Learning with Sparsity: The Lasso and Generalizations (Chapman and Hall, London, 2015).

[17] Y. Kabashima, T. Wadayama, and T. Tanaka, J. Stat. Mech.: Theory Exp. (2009) L09003.
[18] F. Krzakala, M. Mézard, F. Sausset, Y. F. Sun, and L. Zdeborová, Phys. Rev. X 2, 021005 (2012).

[19] M. Mézard and A. Montanari, Information, Physics, and Computation (Oxford University Press, New York, 2009).

[20] M. Mézard, M. Tarzia, and C. Toninelli, J. Phys.: Conf. Ser. 95, 012019 (2008).

[21] D. Sejdinovic and O. Johnson, in 48th Annual Allerton Conference on Communication, Control, and Computing (Allerton) (IEEE, New York, 2010), pp. 998-1003.

[22] T. Kanamori, H. Uehara, and M. Jimbo, J. Stat. Theory Pract. 6, 220 (2012).

[23] A. Sakata, J. Phys. Soc. Jpn. 89, 084001 (2020).

[24] G. Kitagawa, Commun. Stat. - Theory Methods 26, 2223 (1997).

[25] D. D. Lewis and W. A. Gale, in ACM SIGIR Conference on Research and Development in Information Retrieval (ACM/Springer, New York, 1994), pp. 3-12.

[26] D. D. Lewis and J. Catlett, in International Conference on Machine Learning (ICML) (Morgan Kaufmann, Burlington, 1994), pp. $148-156$.

[27] We note the heuristics used in the simulation. When $\rho$ is sufficiently small, such as $\rho=0.005$, BP for the active pooling does not converge sometimes due to the overlapped pools; that is, certain pools are selected several times in the adaptive stage. It is known that rank deficiency can cause the instability of BP. To avoid this problem, we exclude the already existing pool from the candidates in the subsequent stage for the small- $\rho$ case.

[28] R. Kumar and A. Indrayan, Indian Pediatr. 48, 277 (2011).

[29] K. Hajian-Tilaki, Caspian J. Intern. Med. 4, 627 (2013).

[30] M. Mézard and T. Mora, J. Physiol. Paris 103, 107 (2009).

[31] M. Yasuda and K. Tanaka, Phys. Rev. E 87, 012134 (2013). 